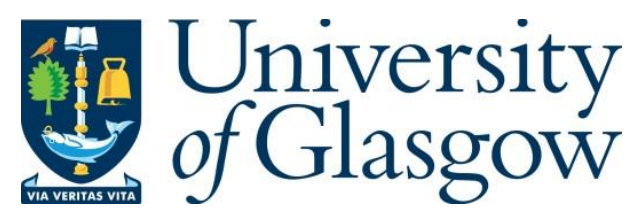

Chang, B., Zhang, L., Li, L., Zhao, G. and Chen, Z. (2019) Optimizing resource allocation in URLLC for real-time wireless control systems. IEEE Transactions on Vehicular Technology, 68(9), pp. 8916-8927.

There may be differences between this version and the published version. You are advised to consult the publisher's version if you wish to cite from it.

http://eprints.gla.ac.uk/190472/

Deposited on: 17 July 2019

Enlighten - Research publications by members of the University of Glasgow http://eprints.gla.ac.uk 


\title{
Optimizing Resource Allocation in URLLC for Real-Time Wireless Control Systems
}

\author{
Bo Chang, Lei Zhang, Liying Li, Guodong Zhao, and Zhi Chen
}

\begin{abstract}
As one of the three main scenarios in the fifth generation (5G) cellular networks, ultra-reliable and low-latency communication (URLLC) can be served as an enabler for real-time wireless control systems. In such a system, the communication resource consumption in URLLC and the control subsystem performance are mutually dependent. To optimize the overall system performance, it is critical to integrate URLLC and control subsystems together by formulating a co-design problem. In this paper, based on uplink transmission, we study the resource allocation problem for URLLC in real-time wireless control systems. The problem is conducted by optimizing bandwidth and transmission power allocation in URLLC and control convergence rate subject to the constraints on communication and control. To formulate and solve the problem, we first convert the control convergence rate requirement into a communication reliability constraint. Then, the co-design problem can be replaced by a regular wireless resource allocation problem. By proving the converted problem is concave, an iteration algorithm is proposed to find the optimal communication resource allocation. Based on that, the optimal control convergence rate can be obtained to optimize overall system performance. Simulation results show remarkable performance gain in terms of spectral efficiency and control cost. Compared with the scheme of satisfying fixed quality-of-service in traditional URLLC design, our method can adjust optimal spectrum allocation to maximize the communication spectral efficiency and maintain the actual control requirement.
\end{abstract}

Index Terms-URLLC, real-time wireless control; co-design; optimal resource allocation.

\section{INTRODUCTION}

As one of the most important communication scenarios in the coming fifth generation $(5 \mathrm{G})$ cellular networks, ultrareliable and low-latency communication (URLLC) is defined as a communication scenario to provide high reliability and low latency end-to-end (E2E) services. URLLC has shown its potential to provide service to real-time wireless control systems, which can obtain significant advantage compared with traditional wired control systems. For instance, the system deployment can be modified or upgraded flexibly while maintaining the commutation quality unaltered. In vehicle technologies, URLLC is one of the key enablers to in-vehicle wireless control, which can significantly reduce financial cost in manufacturing. This further improves fuel efficiency and reduces gas emission to provide timely, reliable and accurate control and an open architecture for new applications [1][2].

B. Chang and Z. Chen are with the National Key Lab. of Science and Technology on Communications, University of Electronic Science and Technology of China (UESTC), Chengdu, 611731, China (e-mail: changb3212@163.com; chenzhi@uestc.edu.cn).

L. Zhang, L. Li, and G. Zhao are with the School of Engineering, University of Glasgow, Glasgow, G12 8QQ, UK (e-mail: Lei.Zhang@glasgow.ac.uk; liyingli0815@gmail.com; gdngzhao@gmail.com).
Furthermore, URLLC is crucial for vehicle-to-anything (V2X) communication, which is essential for the vehicle automatic driving via wireless communications [3][4].

There are some research on wireless control systems, but most of them analyze URLLC (e.g., [5]-[14]) and wireless networked control (e.g., [15]-[20]) separately. From wireless communication perspective, the authors in [21] investigated the achievable channel coding rate for finite blocklength, which establish one of the most important foundations of URLLC design in physical layer. The authors in [22] further discussed the close form expression of the achievable capacity in URLLC, which provides a guideline for the URLLC design in different channel cases, e.g., additive white gaussian noise (AWGN) channel and Rayleigh fading channel. Based on the above research, more works focus on URLLC resource allocation design to maintain the extreme high quality-ofservice (QoS) in URLLC [5]-[12]. For example, the authors in [5] studied the resource allocation for the uplink communications and found that a huge amount of frequency and transmission power are needed to satisfy the extreme high QoS in URLLC. However, all of the research are targeting a latency or reliability constrained communication system without bringing specific control performance (e.g., control cost, stability, or state update rate) into consideration.

From wireless control perspective, the communication latency and reliability are set as random variables caused by communication protocols [15]-[20]. For example, the authors in [15] studied the effect of communication packet loss on the control performance caused by transmission control protocol (TCP) or user datagram protocol (UDP). The authors in [16] further discussed the effect of both time delay and packet loss on the control performance caused by carrier sense multiple access/collision avoidance (CSMA/CA). In [20], the authors provided a survey on the wireless network design for the control systems, where the latency and the reliability were discussed in different communication protocols. The above research indicates that the communication time delay and packet loss results in control performance loss since they enlarge the sample period of the control systems [20]. However, no close form expression is obtained to show exactly how the communication latency and reliability affect the control performance.

The aforementioned work that separately design communication and control subsystems cannot guarantee optimal overall system performance. On the one hand, most of the research on URLLC intend to maintain the extreme high QoS only in wireless communications. On the other hand, the effect of the communication latency and reliability on control 
performance cannot be obtained exactly. In fact, the co-design of the URLLC and real-time wireless control is critical for both communication and control. First, communication resource is extremely limited, especially to meet the ultra high technical requirements for URLLC. A tailored design for a wireless control system can significantly reduce the communication resource consumption. For instance, at some stages when the control performance is not constrained in an extreme high level, the allocated wireless resource can be reduced accordingly. Second, the control performance is determined by both the control sample period and the communication QoS, where the sample period should be adjusted accordingly to optimize the control performance when the communication QoS is different [20].

We notice that there are some research on communicationcontrol co-design [23]-[27]. For instance, in [24], the authors obtained the optimal control sampling period and communication time delay and reliability by simulation analysis based on the existing communication protocols. In [25], we further obtained that different communication QoSs have different effects on control performance throughout the control process by simulation analysis, where the control process can be divided into two stages with different QoS service and the power consumption can be significantly reduced. Furthermore, in [26] and [27], the authors analyzed the control stability with unreliability communications in V2X communications. However, without close form expression indicating the relationship between communication and control by co-design, a high overall system performance cannot be obtained.

This paper aims to optimize the communication resource allocation by joint communication-control design. Taking the uplink transmission as an example, our goal is to find the optimal communication bandwidth, communication power consumption, and control convergence rate ${ }^{1}$ by maximizing the spectral efficiency (SE) while guaranteeing the control requirement. However, the idea can be extended into the downlink transmission scenario straightforwardly. Our problem formulation considers both URLLC QoS and control convergence rate constraints, while such a co-design is still an open problem to be explored. To achieve the goal, a key is to find a method to convert the constraints on the control sub-system into the constraints that the communication subsystem can adopt to solve the optimization problem. The main contributions of this paper are summarized as follows.

- We propose an optimal resource allocation scheme to maximize the SE by communication and control codesign, where both URLLC requirements and control convergence rate requirement are taken into account. The proposed scheme allows us to use optimal resource to support URLLC while guaranteeing the required control performance level.

- We analyze the relationship between the control conver-

\footnotetext{
${ }^{1}$ The main control variables are state sampling period and the control input gain, which jointly affect the control convergence rate. Then, the control convergence rate further affects the control performance, e.g., control cost or control stability. By constraint on control convergence rate, the state sampling period and the control input gain are constrained. Thus, the control convergence rate is considered as the control design in this paper.
}

gence rate requirement and communication requirement on URLLC quality. We find that the lower bound of the communication reliability decreases linearly with the control convergence rate. Then, the requirement on control can be treated as a constraint on the communication reliability, which allows us to convert the co-design problem into a regular communication optimization problem.

- We prove that the formulated optimization problem is concave based on the conversion. Subsequently, we develop an iteration method to find the optimal bandwidth and power allocation.

The rest of this paper is organized as follows. In Section II, the system model is presented. In Section III, the communication-control co-design method is proposed, where the optimal resource allocation problem is formulated with control performance constraint. In Section IV, the relationship between the communication and control subsystems is first discussed, then an iteration method is proposed to obtain the optimal resource allocation in terms of maximizing the communication SE. In Section V, simulation results are provided to show the performance. Finally, Section VI concludes the paper.

\section{SyStem Model With LATENCY AND RELIABILITY CONSTRAINTS}

In this section, we propose the system model by taking communication latency and reliability into consideration. As shown in Fig. 1, we consider a typical centralized real-time wireless communication-control system, where there are $M$ static sensors transmitting sampling signals of the plant state to the base station (BS), the remote controller in the BS processing the sampling signals, and then the BS sending the calculated control command signals to the $M$ corresponding static plants. With the control process continuing, the state of each plant will converge to a preset value. Note that all the notations to be used throughout the paper for communication subsystem and control subsystem are summarized in Table I and II, respectively.

\section{A. Wireless Control Model}

In this subsection, we present the real-time wireless control model considering communication time delay and reliability. Except the inherent control parameters, e.g., the mass and the speed of the plant, the main coefficients that contribute to control performance include sampling period at the sensor and communication QoS [20]. Since we focus on the control convergence rate requirement and communication QoS, constant sampling period is adopted. Then, the continuous control function for the $m$-th plant is given by a linear differential equation as [24]

$$
d \mathbf{x}_{m}(t)=\mathbf{A}_{m} \mathbf{x}_{m}(t) d t+\mathbf{B}_{m} u_{m}(t) d t+d \mathbf{n}_{m}(t),
$$

where $\mathbf{x}_{m}(t)$ is the state of the $m$-th plant, $u_{m}(t)$ is the control input, and $\mathbf{n}_{m}(t)$ is the disturbance caused by additive white gaussian noise (AWGN) with zero mean and variance $\mathbf{R}_{m}$. In addition, $\mathbf{A}_{m}$ and $\mathbf{B}_{m}$ represent the system parameter matrices. To illustrate the system parameters $\mathbf{A}_{m}$ and $\mathbf{B}_{m}$, we 
TABLE I: Summary of Notations for Communication Subsystem

\begin{tabular}{|c|c|c|c|}
\hline$B_{0}$ & Bandwidth of each subcarrier & $R$ & Coverage radius of the base station \\
\hline$C_{m, n}$ & Shannon capacity of the $m$-th sensor at time index $n$ & $T_{t h}$ & Maximum transmission time delay \\
\hline$g_{m}$ & Path-loss of the $m$-th sensor & $T_{m, n}$ & Time resource of the uplink for the $m$-th sensor \\
\hline$h_{m, n}$ & Small scale fading for the $m$-th sensor & $V_{m, n}$ & Channel dispersion for the $m$-th sensor \\
\hline$N_{m, n}$ & Total available number of subcarrier & $\alpha_{m, n}$ & Indicator for packet loss \\
\hline$N_{m}$ & Number of sub-carrier for the $m$-th sensor & $\gamma_{m, n}$ & Received SNR of the $m$-th sensor \\
\hline$l_{m}$ & Distance between the $m$-th plant and the base station & $P_{\max }$ & Maximum of transmission power \\
\hline$M$ & Total number of sensors in the coverage of the base station & $\varepsilon_{m, n}$ & Packet error probability of the uplink for the $m$-th sensor \\
\hline$m$ & Index of the sensors $(1 \leq m \leq M)$ & $\varepsilon_{t h}$ & Maximum packet error probability in communications \\
\hline$N_{0}$ & Variance of the AWGN on each subcarrier at the BS & $\eta_{m}$ & Spectral efficiency of the uplink for the $m$-th sensor \\
\hline$p_{m}$ & Transmission power spectral density & $\lambda_{m}$ & Payload information of the $m$-th sensor at each sample time \\
\hline
\end{tabular}

TABLE II: Summary of Notations for Control Subsystem

\begin{tabular}{|c|c|c|c|}
\hline $\mathbf{A}_{m}$ & System parameter on state in continuous control function & $u_{m, n}^{a}$ & Control input calculated by the actuator \\
\hline $\mathbf{B}_{m}$ & System parameter on input in continuous control function & $u_{m, n}^{c}$ & Feedback parameter calculated by the remote controller \\
\hline$c_{m}$ & Control performance constraint on wireless communications & $u_{m, n}$ & Control parameter for simplify \\
\hline$J_{a v e}$ & Average control cost & $\mathbf{x}_{m}(t)$ & Plant state in continuous control function of the $m$-th plant \\
\hline$N$ & Total sampling time index in control process & $\mathbf{x}_{m, n}$ & Plant state in discrete time control function \\
\hline$n$ & Sample time index & $\Delta_{m}(\cdot)$ & Lyapunov-like function \\
\hline $\mathbf{n}_{m}(t)$ & Disturbance caused by AWGN in continuous control function & $\mathbf{\Phi}_{0}^{m, n}$ & System parameter on input in discrete control function \\
\hline $\mathbf{n}_{m, n}$ & Disturbance in discrete time control function & $\boldsymbol{\Phi}_{1}^{m, n}$ & System parameter on input caused by time delay \\
\hline$\overline{\mathbf{n}}_{m, n}$ & Generalized disturbance & $\boldsymbol{\Omega}_{e_{0}}$ & General system parameter when packet losses \\
\hline $\mathbf{Q}_{m}$ & Given positive definite matrix & $\boldsymbol{\Omega}_{e_{1}}$ & General system parameter when packet transmits successfully \\
\hline $\mathbf{R}_{m}$ & Variance of $\mathbf{n}_{m}(t)$ & $\boldsymbol{\Omega}_{m, d}$ & Generalized system parameter on state \\
\hline$s_{m, n}$ & Sample period of the $m$-th plant at time index $n$ & $\boldsymbol{\Omega}_{m, n}$ & System parameter on state in discrete control function \\
\hline $\bar{s}_{m, n}$ & Idle time before the sampling at time index $n$ & $\xi_{m, n}$ & Generalized plant state \\
\hline$u_{m}(t)$ & Control input in continuous control function & $\rho_{m}$ & Control convergence rate \\
\hline
\end{tabular}

consider a controlled inverted pendulum system as shown in Fig. 2.

Example 1: The $m$-th controlled inverted pendulum system consists of an inverted pendulum and a motorized cart. We have the plant state $\mathbf{x}(t)=\left(c_{t}, \dot{c}_{t}, \theta_{t}, \dot{\theta}_{t}\right)$, where $c_{t}$ represents the cart's position, $\dot{c}_{t}$ represents the cart's velocity, $\theta_{t}$ represents the pendulum's angle, and $\dot{\theta}_{t}$ represents the pendulum's angular velocity. The expression of $\mathbf{A}_{m}$ and $\mathbf{B}_{m}$ consists of the pendulum length $2 l$, the inertia of the pendulum $\Psi$, the friction of the cart $r$, the gravitational acceleration $\phi$, the mass of the pendulum $\lambda$, and the mass of the cart $\Gamma$. By physicalmathematics calculation, $\mathbf{A}_{m}$ and $\mathbf{B}_{m}$ can be expressed as follows, respectively,

$$
\mathbf{A}_{m}=\left(\begin{array}{cccc}
0 & 1 & 0 & 0 \\
0 & \frac{-\left(\Psi+\lambda l^{2}\right) r}{\Psi(\Gamma+\lambda)+\Gamma \lambda l^{2}} & \frac{\lambda^{2} g l^{2}}{\Psi(\Gamma+\lambda)+\Gamma \lambda l^{2}} & 0 \\
0 & 0 & 0 & 1 \\
0 & \frac{-\lambda l r}{\Psi(\Gamma+\lambda)+\Gamma \lambda l^{2}} & \frac{\lambda \phi l(\Gamma+\lambda)}{\Psi(\Gamma+\lambda)+\Gamma \lambda l^{2}} & 0
\end{array}\right)
$$

and

$$
\mathbf{B}_{m}=\left(\begin{array}{c}
0 \\
\frac{\left(\Psi+\lambda l^{2}\right) r}{\Psi(\Gamma+\lambda)+\Gamma \lambda l^{2}} \\
0 \\
\frac{\lambda l}{\Psi(\Gamma+\lambda)+\Gamma \lambda l^{2}}
\end{array}\right) .
$$

To obtain the discrete time control model, we assume that $s_{m, n}$ represents the sample period of the $m$-th plant at time 


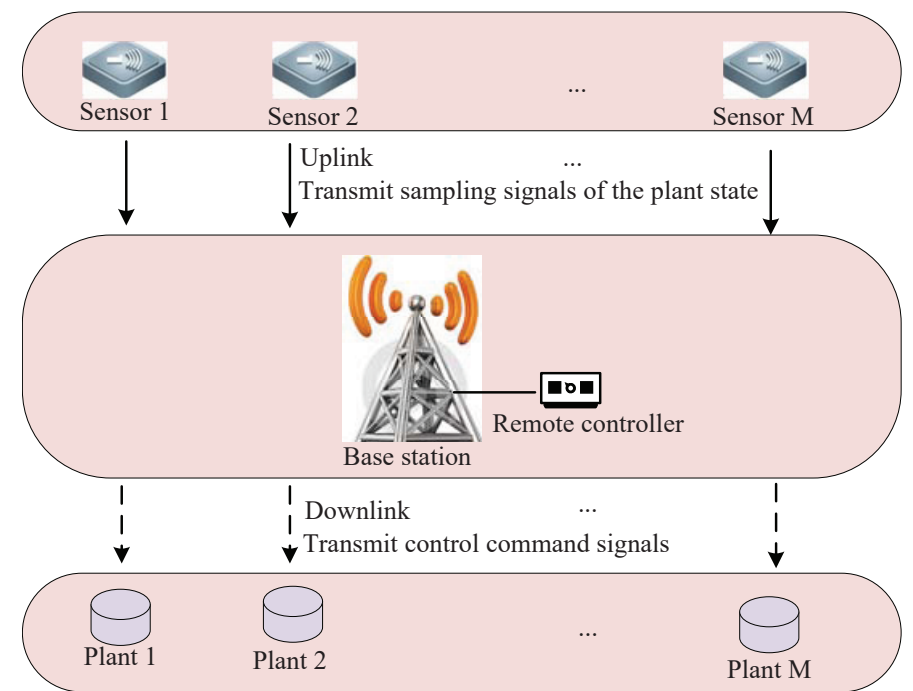

Fig. 1: Real-time wireless control system model.

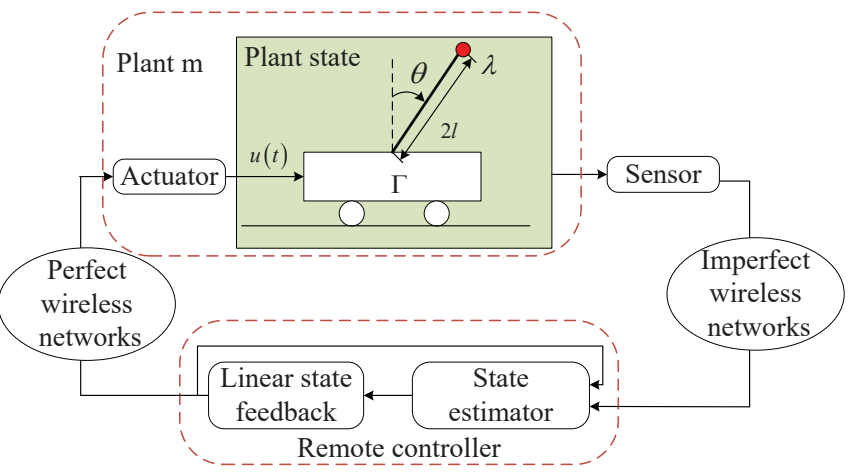

Fig. 2: Wireless control system model for a single plant.

index $n$, which consists of wireless transmission time delay $T_{m, n}$ and an idle period $\bar{s}_{m, n}$. Their relationship can be expressed as

$$
s_{m, n}=\bar{s}_{m, n}+T_{m, n},
$$

where $n=1,2, \cdots, N$ represents the sampling time index in the control process and $N$ is the maximum sampling time index. Then, the discrete time control model with time delay $T_{m, n}$ can be obtained as

$$
\mathbf{x}_{m, n+1}=\boldsymbol{\Omega}_{m, n} \mathbf{x}_{m, n}+\boldsymbol{\Phi}_{0}^{m, n} u_{m, n}+\boldsymbol{\Phi}_{1}^{m, n} u_{m, n-1}+\mathbf{n}_{m, n},
$$

where $\boldsymbol{\Omega}_{m, n}=e^{\mathbf{A} s_{m, n}}, \boldsymbol{\Phi}_{0}^{m, n}=\left(\int_{0}^{\bar{s}_{m, n}} e^{\mathbf{A}_{m, n} t} d t\right) \cdot \mathbf{B}_{m, n}$, and $\boldsymbol{\Phi}_{1}^{m, n}=\left(\int_{\bar{s}_{m, n}}^{s_{m, n}} e^{\mathbf{A}_{m, n} t} d t\right) \cdot \mathbf{B}_{m, n}$. More details about the control model can be referenced in [20].

Assuming $\xi_{m, n}=\left(\begin{array}{ll}\mathbf{x}_{m, n}^{T} & u_{m, n-1}\end{array}\right)^{T}$ is the generalized state, then the control function in (5) can be rewritten as

$$
\xi_{m, n+1}=\boldsymbol{\Omega}_{m, d} \xi_{m, n}+\boldsymbol{\Phi}_{m, d} u_{m, n}+\overline{\mathbf{n}}_{m, n},
$$

where $\overline{\mathbf{n}}_{m, n}=\left(\begin{array}{ll}\mathbf{n}_{m, n}^{T} & 0\end{array}\right)^{T}$ and $\boldsymbol{\Phi}_{m, d}=\left(\begin{array}{l}\boldsymbol{\Phi}_{0}^{m, n} \\ \mathbf{I}\end{array}\right)$. We assume $\boldsymbol{\Omega}_{m, n}=\boldsymbol{\Omega}_{m}$. Then, we have $\boldsymbol{\Omega}_{m, d}=\left(\begin{array}{ll}\boldsymbol{\Omega}_{m} & \boldsymbol{\Phi}_{1}^{m, n} \\ 0 & 0\end{array}\right)$.
Considering the packet loss, we have $\operatorname{Pr}\left\{\alpha_{m, n}=1\right\}=$ $1-\varepsilon_{m, n} \geq 1-\varepsilon_{t h}$ and $\operatorname{Pr}\left\{\alpha_{m, n}=0\right\}=\varepsilon_{m, n}<\varepsilon_{t h}$, where " 1 " means that the packet is successfully transmitted and the control is under closed loop, and " 0 " means that the packet is lost and the control is under open loop. In addition, we assume that the state estimator is perfect, and then a linear feedback $u_{m, n}=\Theta_{m} \xi_{m, n}$ is used. Then, we have the closedloop system in (6) can be rewritten as

$$
\xi_{m, n+1}= \begin{cases}\left(\boldsymbol{\Omega}_{m, d}+\boldsymbol{\Phi}_{m, d} \Theta_{m}\right) \xi_{m, n}+\overline{\mathbf{n}}_{m, n}, & \text { if } \alpha_{m, n}=1 \\ \boldsymbol{\Omega}_{m, d} \xi_{m, n}+\overline{\mathbf{n}}_{m, n}, & \text { if } \alpha_{m, n}=0,\end{cases}
$$

which can be rewritten in a general way as

$$
\xi_{m, n+1}= \begin{cases}\boldsymbol{\Omega}_{e_{1}} \xi_{m, n}+\overline{\mathbf{n}}_{m, n}, & \text { if } \alpha_{m, n}=1 \\ \boldsymbol{\Omega}_{e_{0}} \xi_{m, n}+\overline{\mathbf{n}}_{m, n}, & \text { if } \alpha_{m, n}=0,\end{cases}
$$

where $\boldsymbol{\Omega}_{e_{1}}=\boldsymbol{\Omega}_{m, d}+\boldsymbol{\Phi}_{m, d} \Theta_{m}$ is the parameter of the control system with transmission time delay included in $\boldsymbol{\Omega}_{m, d}$ when the data packet is successfully transmitted, and $\boldsymbol{\Omega}_{e_{0}}=\boldsymbol{\Omega}_{m, d}$ is the parameter of the control system with transmission time delay included in $\boldsymbol{\Omega}_{m, d}$ when the data packet is failed. Furthermore, when there is no transmission time delay, i.e., $d_{m, n}=0$, the expression (5) can be written as

$$
\mathbf{x}_{m, n+1}=\boldsymbol{\Omega}_{m, n} \mathbf{x}_{m, n}+\boldsymbol{\Phi}_{0}^{m, n} u_{m, n}+\mathbf{n}_{m, n} .
$$

Then, the expression (8) can be written as

$$
\mathbf{x}_{m, n+1}=\left\{\begin{array}{l}
\boldsymbol{\Omega}_{e_{1}} \mathbf{x}_{m, n}+\overline{\mathbf{n}}_{m, n}, \text { if } \alpha_{m, n}=1 \\
\boldsymbol{\Omega}_{e_{0}} \mathbf{x}_{m, n}+\overline{\mathbf{n}}_{m, n}, \text { if } \alpha_{m, n}=0,
\end{array}\right.
$$

where $\boldsymbol{\Omega}_{e_{1}}=\boldsymbol{\Omega}_{m, n}+\boldsymbol{\Phi}_{0}^{m, n} \Theta_{m}$ is the parameter of the control system without transmission time delay when the data packet is successfully transmitted, and $\boldsymbol{\Omega}_{e_{0}}=\boldsymbol{\Omega}_{m, n}$ is the parameter of the control system without transmission time delay when the data packet is failed. The above discussion indicates that the proposed presented model in this section can be used regardless of transmission time delay.

\section{B. Wireless Communication Model}

In the rest of this paper, we focus on the uplink from the sensor to the BS, but the derivations can be extended into the downlink transmission straightforwardly. For the convenience of discussion, we assume that the uplink is imperfect that experiences transmission time delay and packet loss, and the downlink is perfect. The scenario with both uplink and downlink experiencing imperfect channel will be considered as a future work. Specifically, $M$ plants are randomly distributed in the coverage of the BS with radius $R$. In addition, each plant is sampled by a corresponding sensor. To avoid interference, we consider orthogonal frequency division multiple access (OFDMA), where each sensor is allocated multiple subcarriers within a given continuous bandwidth, each subcarrier can be allocated to at most one sensor, and the given bandwidths for different sensors are not overlapped. Furthermore, we consider flat fading channel, where the channel gains over different subcarriers for one sensor are approximately identical and perfectly known for the sensor. We assume that the number of allocated bandwidth and transmission duration for the $m$-th sensor at time index $n$ are $N_{m, n}$ and $T_{m, n}$, respectively. Then, 
for the $m$-th sensor at time index $n$, the received signal-tonoise-ratio (SNR) at the BS can be expressed as

$$
\gamma_{m, n}=\frac{\left|h_{m, n}\right|^{2} g_{m} N_{m, n} B_{0} p_{m, n}}{N_{0} N_{m, n} B_{0}}=\frac{\left|h_{m, n}\right|^{2} g_{m, n} p_{m, n}}{N_{0}}
$$

where $h_{m, n}$ is the small scale fading for the $m$-th sensor at time index $n, g_{m}$ is the path-loss, $N_{m, n}$ is the number of allocated bandwidth, $p_{m}$ is the allocated transmission power spectral density for the $m$-th sensor, $B_{0}$ is the separation among subcarriers, and $N_{0}$ is the single-sided noise spectral density. Then, the allocated bandwidth for the $m$-th sensor is $B_{m, n}=B_{0} N_{m, n}$. Based on the received SNR, the Shannon capacity can be expressed as

$$
C_{m, n}=\log \left(1+\gamma_{m, n}\right) .
$$

In URLLC, with the constraints on utra-reliability and lowlatency, the blocklength is short and small packet size is adopted [7]. In addition, the size of payload data transmitted by sensor in control system is usually small (e.g., about 100 bits [20]), which is suitable to be used in URLLC scenario. In such a scenario, the impact of decoding error cannot be ignored. We assume that channel dispersion $V_{m, n}$ is adopted to represent the capacity loss caused by the transmission error, which can be expressed as [22]

$$
V_{m, n}=(\log e)^{2}\left(1-\frac{1}{\left(1+\gamma_{m, n}^{2}\right)}\right) \text {. }
$$

When SNR is higher than $5 \mathrm{~dB}$, we have $V_{m, n} \approx(\log e)^{2}$, which is very accurate [7]. When $V_{m, n}<(\log e)^{2}$, we can obtain a lower bound of the achievable rate with channel dispersion by substituting $V_{m, n}=(\log e)^{2}$. Thus, in the following of this paper, we adopt

$$
V_{m, n}=(\log e)^{2} .
$$

For the $m$-th plant, the available uplink rate with finite block length is the uplink capacity eliminating the error bits that are introduced by channel dispersion, which can be expressed as

$$
\begin{aligned}
& R_{m, n}=C_{m, n}- \\
& \sqrt{\frac{V_{m, n}}{N_{m, n} T_{m, n} B_{0}}} f_{Q}^{-1}\left(\varepsilon_{m, n}\right)+\frac{\log \left(N_{m, n} T_{m, n} B_{0}\right)}{2 N_{m, n} T_{m, n} B_{0}},
\end{aligned}
$$

where $\frac{\log \left(N_{m, n} T_{m, n} B_{0}\right)}{2 N_{m, n} T_{m, n} B_{0}}$ is the approximation of the remainder terms of order $\log \left(N_{m, n} T_{m, n} B_{0}\right) /\left(N_{m, n} T_{m, n} B_{0}\right)$, and $f_{Q}^{-1}(\cdot)$ is the inverse of the Q-function. Then, the packet error probability can be expressed as (16) on the top of this page.

For the above channel model, it consists of path-loss and small scale fading. According to [28], the path-loss $g_{m}$ can be expressed as

$$
g_{m_{[d B]}}=-128.1-37.6 \lg \left(l_{m}\right),
$$

where $l_{m}$ is the distance between the $m$-th plant and the BS with unit $\mathrm{km}$ and is larger than $0.035 \mathrm{~km}$ [28]. The small-scale fading $h_{m, n}$ follows Rayleigh distribution with mean zero and variance $\sigma_{0}^{2}=1$. In addition, small-scale fading is constant within coherence time, which is larger than the maximum endto-end (E2E) time delay. Thus, we consider quasi-static fading channel, which is constant for each uplink subcarrier within a frame [29].

From (12), (14) (16), and (15), we can obtain the SE of the uplink for the $m$-th plant at time index $n$, which can be expressed as

$$
\eta_{m, n}=\frac{\lambda_{m}}{N_{m, n} B_{0}}\left(1-\varepsilon_{m, n}\right)
$$

where $\lambda_{m}$ is the desired payload transmitted by the sensor, and the SE means successful decoding bits at the BS per bit. In this paper, we intend to obtain the uplink optimal wireless resource allocation by maximizing SE in (18).

\section{Communication-Control Co-Design}

Our goal is to maximize the communication SE and maintain good overall system performance. Thus, in the following of this section, we first discuss the constraints from the perspectives of communication and control, respectively. Then, we formulate the co-design problem based on the constraints.

\section{A. Communication Constraint}

The main constraints from communication are the limited wireless resource and URLLC QoS. We assume that the total available transmission power and bandwidth without reuse among sensors for each sensors are $p^{\max }$ and $B^{\max }$ at each time index $n$, respectively, where the constraints can be expressed as

$$
N_{m, n} B_{0} p_{m, n} \leq p^{\max }
$$

and

$$
B_{m, n}=N_{m, n} B_{0} \leq B^{\max } .
$$

Based on the constraint of the reliability in URLLC, the successful transmission probability can be expressed as

$$
\operatorname{Pr}\left\{\alpha_{m, n}=1\right\}=1-\varepsilon_{m, n} \geq 1-\varepsilon_{t h},
$$

and the failed transmission probability can be expressed as

$$
\operatorname{Pr}\left\{\alpha_{m, n}=0\right\}=\varepsilon_{m, n}<\varepsilon_{t h},
$$

where $\varepsilon_{t h}$ is the packet error probability bounded by the URLLC QoS requirement. Furthermore, the communication time delay should also be bounded by the URLLC QoS requirement. Then, we have

$$
T_{m, n} \leq T_{t h}
$$

We assume that the sizes of payload $\lambda_{m}$ for each sensor are identical, which can be rewritten as $\lambda$. Then, for given $\lambda$, we have $N_{m, n} T_{m, n} B_{0} R_{m, n}=\lambda$. Then, (16) and (18) can be rewritten as

$$
\varepsilon_{m, n}=f_{Q}\left(\frac{N_{m, n} T_{m, n} B_{0} C_{m, n}-\lambda+\left(\log N_{m, n} T_{m, n} B_{0}\right) / 2}{(\log e) \cdot \sqrt{N_{m, n} T_{m, n} B_{0}}}\right),
$$

and

$$
\eta_{m, n}=\frac{\lambda}{N_{m, n} B_{0}}\left(1-\varepsilon_{m, n}\right)
$$




$$
\begin{aligned}
\varepsilon_{m, n} & =f_{Q}\left(\frac{N_{m, n} T_{m, n} B_{0} C_{m, n}-N_{m, n} T_{m, n} B_{0} R_{m, n}+\left(\log N_{m, n} T_{m, n} B_{0}\right) / 2}{\sqrt{N_{m, n} T_{m, n} B_{0} V_{m, n}}}\right) \\
& =f_{Q}\left(\frac{N_{m, n} T_{m, n} B_{0} C_{m, n}-N_{m, n} T_{m, n} B_{0} R_{m, n}+\left(\log N_{m, n} T_{m, n} B_{0}\right) / 2}{(\log e) \cdot \sqrt{N_{m, n} T_{m, n} B_{0}}}\right)
\end{aligned}
$$

where $f_{Q}(\cdot)$ is the Q-function.

\section{B. Control Constraint}

In this paper, we consider the control convergence rate $\rho_{m}$ as the constraint from the control aspect, where the sampling period is fixed and the inherent parameters of the plant are physical reality and change only when the plants are different. Given physical control system, the main control variables that can be optimized are state sampling period $s_{m, n}$ and the control input gain $\Theta_{m}$, which jointly affect the control convergence rate $\rho_{m}$. Then, the control convergence rate further affects the control performance, e.g., control cost or stability. By constraint on $\rho_{m}$, the state sampling period $s_{m, n}$ and the control input gain $\Theta_{m}$ are directly constrained. In other words, that the relationship between control convergence rate and wireless resource allocation indicates the relationship between the main control variables and wireless resource allocation. Thus, the co-design of this paper focuses on control convergence rate and wireless resource allocation.

To obtain the constraint on the control convergence rate, we consider Lyapunov-like function for each plant, which can be expressed as [32]

$$
\Delta_{m}\left(\xi_{m}\right)=\xi_{m}^{T} \mathbf{Q}_{m} \xi_{m}
$$

where $\mathbf{Q}_{m}$ is a given positive definite matrix. The requirement for the Lyapunov-like function is that these functions should decrease at given rates $\rho_{m}<1$ during the control process, which means that the control process guarantees the plant state decreasing to the pre-set point. However, affected by the control perturbation and stochastic communication coefficients, the Lyapunov-like function is random. Thus, for any possible value of the current plant states $\xi_{m, n}$, the Lyapunovlike functions needs to satisfy [32]

$$
\mathbb{E}\left[\Delta_{m}\left(\xi_{m, n+1}\right) \mid \xi_{m, n}\right] \leq \rho_{m} \Delta_{m}\left(\xi_{m, n}\right)+\operatorname{Tr}\left(\mathbf{Q}_{m} \mathbf{R}_{m}^{\prime}\right)
$$

where $\mathbb{E}[\cdot]$ represents the expectation operator and $\mathbf{R}_{m}^{\prime}=$ $\left(\begin{array}{ll}\mathbf{R}_{m} & 0\end{array}\right)$. The control convergence is guaranteed with $\rho_{m}<1$, which has been discussed in [32]. We conclude it by the following Lemma.

Lemma 1 (Control Convergence Lemma): If (27) holds for each time step $n=0,1,2, \ldots, N$, then by taking the expectation at both sides and by iterating backwards we can obtain

$$
\begin{aligned}
\mathbb{E}\left[\Delta_{m}\left(\xi_{m, N}\right)\right] & \leq \rho_{m} \mathbb{E}\left[\Delta_{m}\left(\xi_{m, N-1}\right)\right]+\operatorname{Tr}\left(\mathbf{Q}_{m} \mathbf{R}_{m}^{\prime}\right) \\
& \leq \ldots \\
& \leq \rho_{m}^{N} \mathbb{E}\left[\Delta_{m}\left(\xi_{m, 0}\right)\right]+\sum_{n=0}^{N-1} \rho_{m}^{n} \operatorname{Tr}\left(\mathbf{Q}_{m} \mathbf{R}_{m}^{\prime}\right)
\end{aligned}
$$

Since the sum in (28) converges with $\rho_{m}<1$, the second moments of the plant states decay exponentially, which is bounded by $\operatorname{Tr}\left(\mathbf{Q}_{m} \mathbf{R}_{m}^{\prime}\right) /\left(1-\rho_{m}\right)$.

\section{Problem Formulation}

According to the constraints from the communication and control aspects, we formulate the original communicationcontrol co-design problem as

$$
\max _{N_{m, n}, p_{m, n}, \rho_{m}} \eta=\sum_{m=1}^{M} \sum_{n=1}^{N} \eta_{m, n}
$$

s.t.

$\varepsilon_{m, n} \leq \varepsilon_{t h}$,

$N_{m} p_{m, n} \leq p^{\max }$,

$B_{m, n} \leq B^{\max }$

$T_{m, n} \leq T_{t h}$,

$\mathbb{E}\left[\Delta_{m, n}\left(\xi_{m, n+1}\right) \mid \xi_{m, n}\right] \leq \rho_{m} \Delta_{m}\left(\xi_{m, n}\right)+\operatorname{Tr}\left(\mathbf{Q}_{m} \mathbf{R}_{m}^{\prime}\right)$.

In this problem, we intend to maximize the communication SE with optimal wireless resource allocation in URLLC and control convergence rate. More importantly, we jointly consider the control constraint on the control convergence rate $\rho_{m}$ and communication constraint on URLLC QoS, which is difficult to deal with in the proposed communication-control co-design since the control constraint (29f) seems independent with rest of the communication constraint terms in (29). However, the control constraint on the control convergence rate $\rho_{m}$ can affect and is determined by the communication QoS actually. In the next section, we will deal with the problem in (29) in details.

\section{Optimal Resource Allocation for The PROPOSED CO-DESIGN}

In this section, we first explore the relationship between control and communication. Then, we discuss the solution for the problem in (29).

\section{A. Relationship Between Control and Communication}

From (8), we can obtain that the plant state $\xi_{m, n}$ in (8) is determined by the control parameters and packet transmission. Then, the expression $\mathbb{E}\left[\Delta_{m}\left(\xi_{m, n+1}\right) \mid \xi_{m, n}\right]$ depends on the packet transmission probability. From (8), we can obtain that 
the Lyapunov-like function can be expressed as

$$
\begin{aligned}
\mathbb{E}\left[\Delta_{m}\left(\xi_{m, n+1}\right) \mid \xi_{m, n}\right] & =\operatorname{Pr}\left\{\alpha_{m, n}=1\right\} \xi_{m, n}^{T} \boldsymbol{\Omega}_{e_{1}}^{T} \mathbf{Q}_{m} \boldsymbol{\Omega}_{e_{1}} \xi_{m, n} \\
& +\operatorname{Pr}\left\{\alpha_{m, n}=0\right\} \xi_{m, n}^{T} \boldsymbol{\Omega}_{e_{0}}^{T} \mathbf{Q}_{m} \boldsymbol{\Omega}_{e_{0}} \xi_{m, n} \\
& +\operatorname{Tr}\left(\mathbf{Q}_{m} \mathbf{R}_{m}^{\prime}\right)
\end{aligned}
$$

which indicates that the communication reliability can affect the control Lyapunov-like function directly. Substituting (30) into (29f), we can obtain

$$
\operatorname{Pr}\left\{\alpha_{m, n}=1\right\} \geq \frac{\xi_{m, n}^{T}\left(\boldsymbol{\Omega}_{e_{0}}^{T} \mathbf{Q}_{m} \boldsymbol{\Omega}_{e_{0}}-\rho_{m} \mathbf{Q}_{m}\right) \xi_{m, n}}{\xi_{m, n}^{T}\left(\boldsymbol{\Omega}_{e_{0}}^{T} \mathbf{Q}_{m} \boldsymbol{\Omega}_{e_{0}}-\boldsymbol{\Omega}_{e_{1}}^{T} \mathbf{Q}_{m} \boldsymbol{\Omega}_{e_{1}}\right) \xi_{m, n}}
$$

where $\xi_{m, n} \neq 0$.

From (31), we can obtain the relationship between the control requirement on the control convergence rate $\rho_{m}$ and communication reliability requirement $\operatorname{Pr}\left\{\alpha_{m, n}=1\right\}$. Here, the lower bound of the communication reliability decreases monotonically with $\rho_{m}$. This is reasonable since when $\rho_{m}$ is small, the plant state updates smoothly, which leads to good control performance [15]. In summary, small $\rho_{m}$ means good control performance and needs high communication reliability to maintain the control performance. On the contrary, large $\rho_{m}$ means the loss in control performance and does not need high communication reliability to maintain the control performance. Furthermore, from (10) and (31), we can obtain that the proposed relationship also works in the scenario where there is no transmission time delay, straightforwardly.

Let

$$
c_{m}=\sup _{y \in \mathbb{R}^{n}, y \neq 0} \frac{y^{T}\left(\boldsymbol{\Omega}_{e_{0}}^{T} \mathbf{Q}_{m} \boldsymbol{\Omega}_{e_{0}}-\rho_{m} \mathbf{Q}_{m}\right) y}{y^{T}\left(\boldsymbol{\Omega}_{e_{0}}^{T} \mathbf{Q}_{m} \boldsymbol{\Omega}_{e_{0}}-\boldsymbol{\Omega}_{e_{1}}^{T} \mathbf{Q}_{m} \boldsymbol{\Omega}_{e_{1}}\right) y}
$$

represent the supremum of the right-hand term in (31). According to Appendix A, we can obtain the optimal $c_{m}$.

Based on the above discussion, we can obtain the following theorem about the relationship between control and communication.

Theorem 1: In real-time wireless control systems, the communication reliability is actually determined by the control performance constrained by the requirement of control convergence rate, rather than the suggested reliability in communications, i.e., being bounded by $\varepsilon_{t h}$. Their relationship can be expressed as

$$
\operatorname{Pr}\left\{\alpha_{m, n}=1\right\} \geq c_{m}^{*}
$$

Based on Theorem 1, we can obtain the following property.

Property 1: According to the relationship between the control performance constraint $c_{m}^{*}$ and the reliability requirement $1-\varepsilon_{t h}$ in URLLC QoS, the actual resource consumption for URLLC in real-time wireless control system can be divided into the following three cases.

- Case A: When $c_{m}^{*}>1-\varepsilon_{t h}$, the actual resource consumption is higher than the bound in the traditional URLLC resource allocation.

- Case B: When $c_{m}^{*}=1-\varepsilon_{t h}$, the actual resource consumption is equal to the bound in the traditional URLLC resource allocation.
- Case C: When $c_{m}^{*}<1-\varepsilon_{t h}$, the actual resource consumption is lower than the bound in the traditional URLLC resource allocation.

In the following of this section, we solve the optimal problem in (29). First, we obtain the optimal communication resource allocation with constraint on control convergence rate. Then, the optimal control convergence rate can be obtained.

\section{B. Optimal Resource Allocation}

In this subsection, we first convert the optimal problem into a solvable problem. Then, we develop an algorithm to obtain the solution for optimal resource allocation.

1) Problem Conversion: Though the state update of the control is relevant in different time index $n$, the wireless resource allocation to guarantee the control requirement is independent. In addition, the available resource, i.e., the transmission power and bandwidth, is independent among different sensors. Thus, we can drop the time indices $n$ and decompose Problem (29) into $M$ subproblems. Furthermore, the constraint on communication reliability in (29b) should be replaced by the relationship in Theorem 1. Thus, (29) can be rewritten as

$$
\begin{aligned}
& \text { s.t. } \\
& \varepsilon_{m} \leq 1-c_{m}^{*}, \\
& N_{m} p_{m} \leq p^{\max }, \\
& B_{m} \leq B^{\max }, \\
& T_{m} \leq T_{t h},
\end{aligned}
$$$$
\max _{N_{m}, p_{m}} \eta_{m}=\frac{\lambda}{N_{m}}\left(1-\varepsilon_{m}\right)
$$

where the control convergence rate $\rho_{m}$ is omitted in the optimal variables since the expression (32) and the constraint in (34b) indicate that $\rho_{m}$ can be obtained by $\varepsilon_{m}=1-c_{m}^{*}$. The optimal control convergence rate $\rho_{m}$ will be discussed later in the next subsection.

Our goal is to maximize the wireless SE by optimal resource allocation, and meanwhile cause less resource consumption. To achieve this goal in solving (34), the time delay should be long enough, i.e.,

$$
T_{m}=T_{t h}
$$

which is because large time domain resource can reduce other resource consumption. Then, we have

$$
\begin{aligned}
\varepsilon_{m} & =f_{Q}\left(\frac{N_{m} T_{t h} B_{0} C_{m}-\lambda+\log \left(N_{m} T_{t h} B_{0}\right) / 2}{(\log e) \sqrt{N_{m} T_{t h} B_{0}}}\right) \\
& \leq 1-c_{m}^{*} .
\end{aligned}
$$

Then, (34) can be rewritten as

$$
\begin{aligned}
& \max _{N_{m}, p_{m}} \frac{\lambda}{N_{m}}\left(1-\varepsilon_{m}\right) \\
& \text { s.t. } \\
& (34 c),(34 d),(36) .
\end{aligned}
$$

This is the final expression of the problem formulation. Next, we focus on the solution to this problem. 
2) Problem Solution: In the sequel, we propose an iteration algorithm to find the global optimal solution of problem (37). Before that, we need the following properties about $\eta_{m}$.

Property 2: Given $N_{m}, \eta_{m}$ decreases monotonically with $p_{0}$.

Proof. It is easy to obtain that $f_{Q}(\cdot)$ in (36) decreases monotonically with $p_{0}$, Then, $\varepsilon_{m}$ increases monotonically with $p_{m}$. Finally, $\eta_{m}$ decreases monotonically with $p_{m}$.

Property 3: Given $p_{m}$, (37a) is a concave function with respect to $N_{m}$.

Proof. See Appendix B.

Based on Property 2 and $\mathbf{3}$, we propose an iterative algorithm to find the optimal solution for problem (37). Given $p_{m}$, the optimal values of $k_{m}$ that maximizes (37) can be found via bisection method. By searching $k_{m}$, the optimal and minimum value of $p_{m}$ can be obtained by (36). Thereby, we can obtain the optimal resource allocation to maximize the communication SE in (37). The details of the algorithm are provided in Algorithm 1.

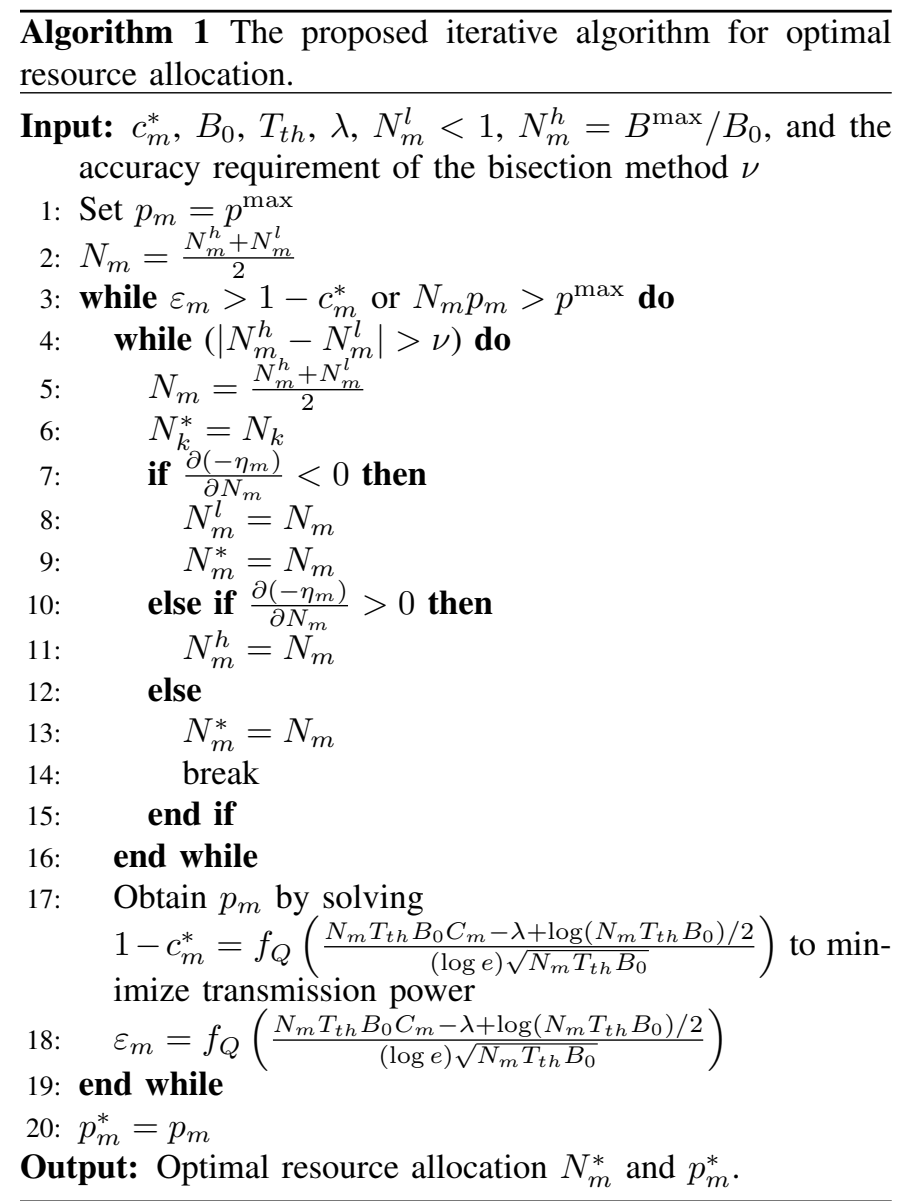

\section{Optimal Control Convergence Rate}

Once we obtain the maximum communication SE in (34), the communication reliability $\left(1-\varepsilon_{m}\right)$ can be obtained accordingly. Then, we can obtain the optimal control convergence rate $\rho_{m}^{*}$ to minimize control cost by solving the following equation

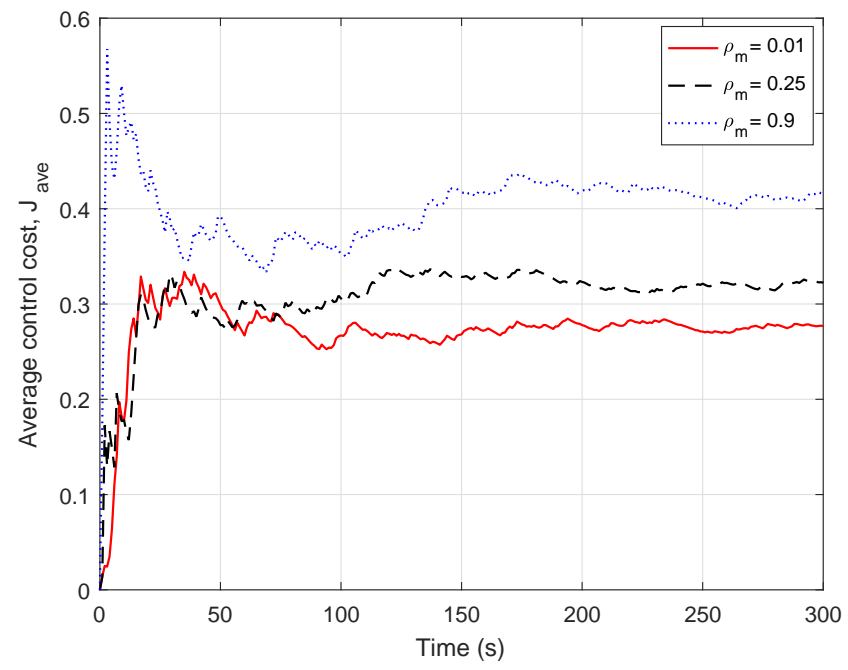

Fig. 3: Average control cost with time increasing.

$$
\begin{aligned}
& \operatorname{Pr}\left\{\alpha_{m, n}=1\right\}=1-\varepsilon_{m}=c_{m}^{*} \\
& =\frac{\xi_{m, n}^{T}\left(\boldsymbol{\Omega}_{e_{0}}^{T} \mathbf{Q}_{m} \boldsymbol{\Omega}_{e_{0}}-\rho_{m} \mathbf{Q}_{m}\right) \xi_{m, n}}{\xi_{m, n}^{T}\left(\boldsymbol{\Omega}_{e_{0}}^{T} \mathbf{Q}_{m} \boldsymbol{\Omega}_{e_{0}}-\boldsymbol{\Omega}_{e_{1}}^{T} \mathbf{Q}_{m} \boldsymbol{\Omega}_{e_{1}}\right) \xi_{m, n}} .
\end{aligned}
$$

Thereby, we can obtain an optimal overall system performance.

\section{Simulations Results}

In this section, we provide simulation results to demonstrate the performance of the proposed method, where the system models are the same as shown in Fig. 1 and Fig. 2. In the following of this section, we first illustrate the control performance. Then, we discuss the optimal resource allocation to maximize the communication $\mathrm{SE}$.

\section{A. Control Performance}

From the perspective of real-time wireless control, since $M$ plants are independent in communication-control co-design, we assume that only two plants, i.e., $M=2$ plants, are considered in the simulations. For simplicity, we assume that both plants have the identical dynamics, where assume that $\Omega_{e_{0}}=1.5$ and $\Omega_{e_{1}}=0.5$. We further assume that the given positive definite weight matrix is $\mathbf{Q}_{m}=1$, the variance of the disturbance matrix $\mathbf{n}_{m}(t)$ is 1, i.e., $\mathbf{R}_{m}=1$, and the sample period is $s_{m, n}=100 \mathrm{~ms}$. In addition, we adopt the average control cost to evaluate the control performance [30], which can be expressed as

$$
J_{\text {ave }}=\frac{1}{N} \Sigma_{n=1}^{N} x_{m, n}^{2},
$$

where $N=T / s_{m, n}$ is adopted, and $T$ is the total time of the control process.

Fig. 3 shows the average control cost $J_{\text {ave }}$ with control time increasing, where we consider three different decreasing rate $\rho_{m}$, i.e., $0.01,0.25$, and 0.9 . From the figure, all the curves increase at the initial time. This is because the control 


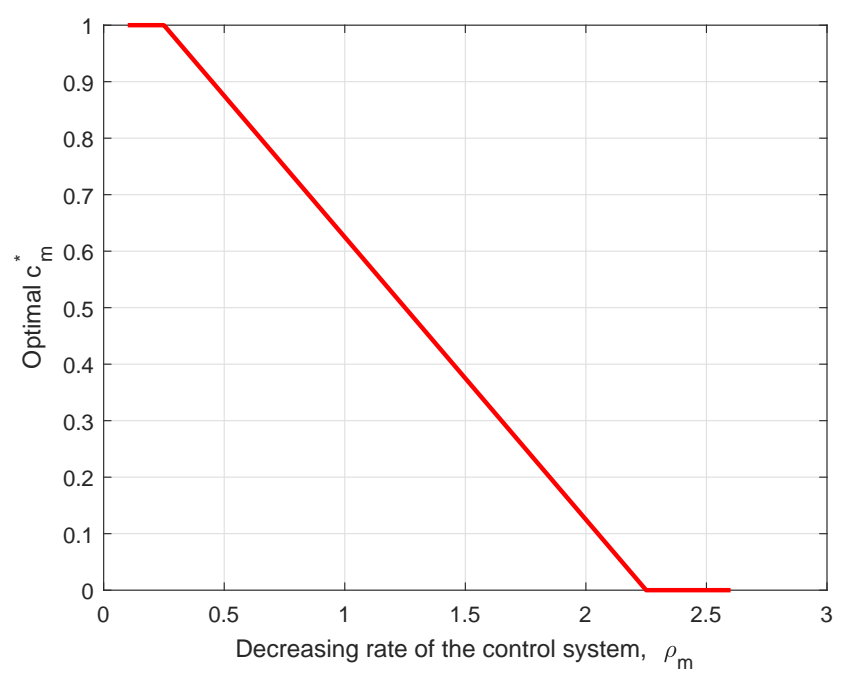

Fig. 4: Optimal $c_{m}^{*}$ with different control convergence rate $\rho_{m}$.

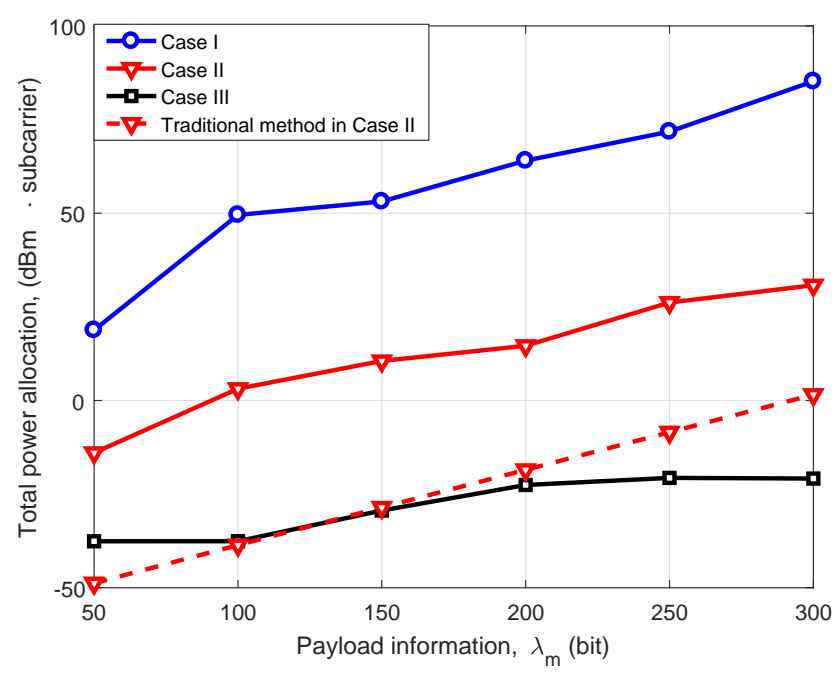

(a) The total resource allocation with different payload information $\lambda_{m}$

Fig. 5: The optimal resource allocation with different payload information $\lambda_{m}$ (con't)

process is performed before the state returns to the pre-set point, where the plant state update leads to the increasing of $J_{\text {ave }}$. Furthermore, as the control time increasing, when $\rho_{m}$ is small, i.e., $\rho_{m}=0.01$, the curve has a little drop and reaches to a low approximative horizontal line smoothly. However, when $\rho_{m}$ is large, i.e., $\rho_{m}=0.9$, the curves have a little drop and reach to a high approximative horizontal line roughly. This is reasonable since the plant state update is smaller when the plant turns to stable than that at the start phase, which leads to the average control cost $J_{\text {ave }}$ has a little drop and reach to an approximative horizontal line. These phenomenons indicate that small decreasing rate $\rho_{m}$ leads to smooth state updating, where we can obtain small average control cost $J_{\text {ave }}$ updating. When $\rho_{m}$ is large, the state updating is rough, which leads to large average control cost $J_{\text {ave }}$ updating. In addition, when $\rho_{m}$ is small, i.e., $\rho_{m}=0.01$, the smooth state updating leads

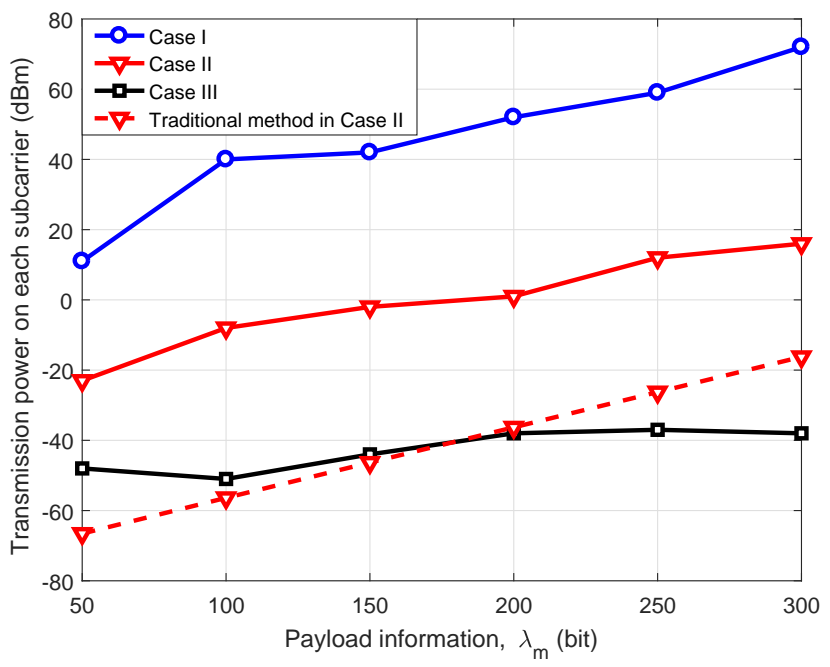

(b) The optimal power allocation with different payload information $\lambda_{m}$

Fig. 5: The optimal resource allocation with different payload information $\lambda_{m}$ (con't)

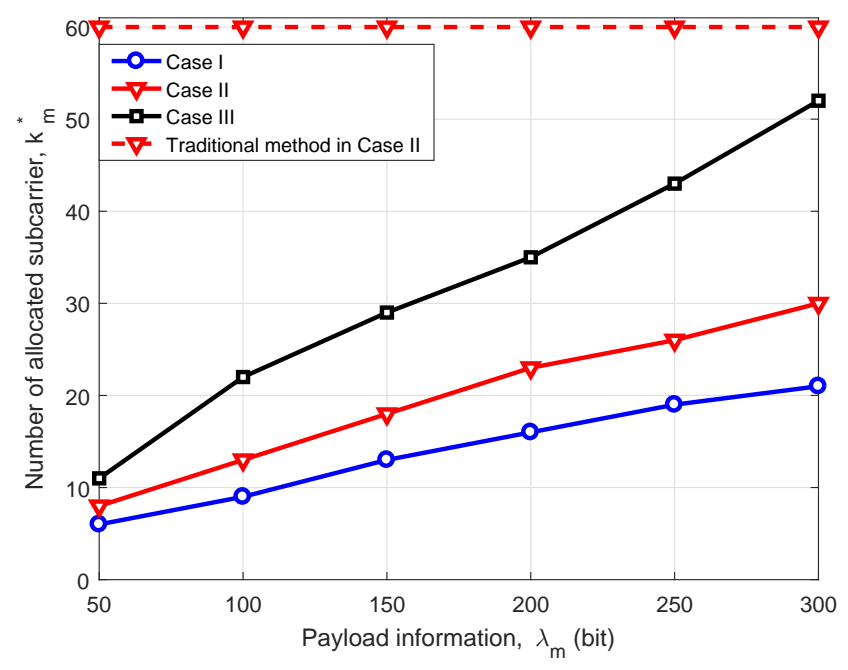

(c) The optimal subcarrier allocation with different payload information $\lambda_{m}$

Fig. 5: The optimal resource allocation with different payload information $\lambda_{m}$.

to low the average control cost as the time is large enough, which means that small decreasing rate has better control performance than large decreasing rate.

Fig. 4 shows the relationship between the optimal $c_{m}^{*}$ in (32) when decreasing rate $\rho_{m}$ is different. From the figure, the curve decreases monotonically with $\rho_{m}$ from $c_{m}^{*}=1$ to $c_{m}^{*}=0$, which means that small $\rho_{m}$ results in high constraint on the communication reliability. This is reasonable since high reliability can guarantee smooth decrease of the plant state from control perspective, which leads to small decreasing rate $\rho_{m}$ and small average control cost in Fig. 3.

From the above control results, we can obtain that Property 1 can be redescribed based on the different values of control 


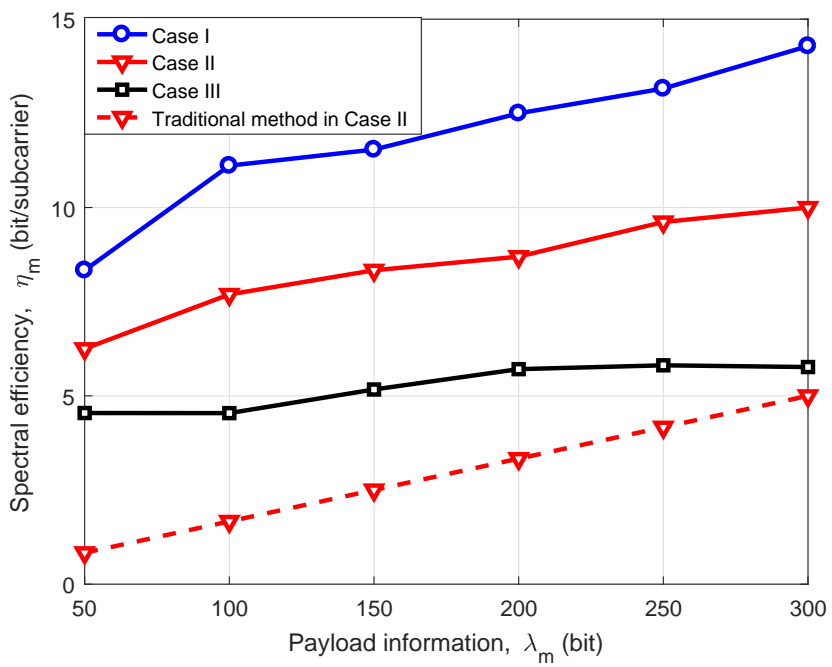

Fig. 6: The spectral efficiency with different payload information $\lambda_{m}$.

decreasing rate $\rho_{m}$, where $c_{m}^{*}\left(\rho_{t h}\right)=1-\varepsilon_{t h}$. Then the three cases in Property 1 can be described as follows.

- Case I: $\rho_{m}<\rho_{t h}$. In this case, $\rho_{m}<\rho_{t h}$ means that $c_{m}^{*}>c_{m}^{*}\left(\rho_{t h}\right)=1-\varepsilon_{t h}$, where the allocated resource in traditional URLLC is failed in guaranteeing the required control performance and more wireless resources are needed.

- Case II: $\rho_{m}=\rho_{t h}$. In this case, $\rho_{m}=\rho_{t h}$ means that $c_{m}^{*}=c_{m}^{*}\left(\rho_{t h}\right)=1-\varepsilon_{t h}$, where the allocated resource in traditional URLLC is just enough in guaranteeing the required control performance and more wireless resource are needed.

- Case III: $\rho_{m}>\rho_{t h}$. In this case, $\rho_{m}>\rho_{t h}$ means that $c_{m}^{*}<c_{m}^{*}\left(\rho_{t h}\right)=1-\varepsilon_{t h}$, where the allocated resource in traditional URLLC is more than that needed in guaranteeing the required control performance and wireless resource waste is presented.

In the following of this section, we discuss the communication performance of our proposed method. Furthermore, we use $c_{m}^{*}=1-10^{-9}, c_{m}^{*}=1-10^{-5}$, and $c_{m}^{*}=1-10^{-3}$ to discuss the communication performance in the above three cases, respectively.

\section{B. Communication Performance}

From the perspective of wireless communication, we assume that the bandwidth of each subcarrier is $1 \mathrm{kHz}$, the single-sided noise spectral density is $-174 \mathrm{dBm} / \mathrm{Hz}$, and the distance between the base station and the plants is 100 $\mathrm{m}$. For the URLLC, the maximum packet transmission error probability is $\varepsilon_{t h}=10^{-5}$, the maximum transmission time delay for the uplink is $T_{t h}=0.5 \mathrm{~ms}$, and the maximum number of allocation subcarrier is 60 . In addition, we consider the traditional method only considering communication aspect in [6] for comparison, where the authors intended to minimize transmission power.
Fig. 5 demonstrates the wireless resource allocation when payload information $\lambda_{m}$ is different. Fig. 5(a) shows the total power allocation. From the figure, the total resource allocation (i.e., multiplying transmission power by frequency bandwidth) increases with communication reliability increasing from Case III to Case I. This is reasonable since high reliability needs more communication resource to support. Fig. 5(b) and Fig. 5(c) show the allocated transmission power on each subcarrier and the allocated number of subcarrier for the $m$-th sensor. From Fig. 5(a), Fig. 5(b) and Fig. 5(c), both the allocated subcarriers and allocated transmission power of the proposed method increase monotonically with $\lambda_{m}$, which means that more payload information needs more resources for transmission. From Fig. 5(b), the allocated transmission power on each subcarrier of the proposed method increases with communication reliability increasing from Case III to Case I. However, from Fig. 5(c), the allocated subcarriers of the proposed method decrease with communication reliability increasing from Case III to Case I. This is reasonable since the proposed method in Algorithm 1 leads to an increasing power allocation from Case III to Case I, which further leads to the subcarrier allocation reducing from Case III to Case I while maintaining total resource allocation increasing from Case III to Case I as shown in Fig. 5(a). In summary, the higher control performance needs more communication resources. In addition, compared with the traditional method in [6], the number of the allocated subcarrier of the proposed method is significantly reduced. For instance, the number of the allocated subcarrier is reduced from 60 to 8 when the payload information is 50 bits in Fig. 5(c), while the allocated transmission power on each subcarrier of the proposed method in Fig. 5(b) is increased from $-65 \mathrm{dBm}$ to $-22 \mathrm{dBm}$, and the total allocated transmission power of the proposed method in Fig. 5(a) is increased from $-50 \mathrm{dBm}$ to $-20 \mathrm{dBm}$. This is reasonable since the traditional method aims to minimize the transmission power with maximum allocated bandwidth, which leads to lower total power allocation for the traditional method as shown in Fig. 5(a).

Fig. 6 shows the spectral efficiency when payload information $\lambda_{m}$ is different. From the figure, it is easy to obtain that all the curves increase with the payload information increasing. In addition, spectral efficiency increases with the communication reliability increasing from Case III to Case I. This is reasonable since the allocated subcarriers decrease with communication reliability increasing from Case III to Case I in Fig. 5(c), which leads to spectral efficiency increasing. Furthermore, compared with traditional method in Case II, the proposed method has larger spectral efficiency, i.e., at most $700 \%$ spectral efficiency performance increasing when payload information is 50 bits. This is reasonable since our method is optimal in maximizing SE.

\section{Conclusions}

In this paper, we proposed an optimal resource allocation scheme to maximize the communication uplink spectral efficiency in URLLC for real-time wireless control systems. In this scheme, we considered the URLLC service should 
satisfy the requirement on control convergence rate, which is formulated as a communication-control co-design problem. To solve the hybrid problem, the control requirement was converted into a constraint on the wireless communication reliability. Then, the hybrid optimization problem can be replaced by a regular wireless resource allocation problem. We proved that the converted problem is concave and an iteration algorithm is developed to obtain the optimal solution. Based on that, the optimal control convergence rate can be obtained. The simulation results showed that the proposed method achieves the maximum spectral efficiency while maintaining the control performance. The proposed co-design approach established a theoretic foundation for the URLLC serviced real-time wireless control system performance analysis and algorithm design.

\section{APPENDIX A}

This appendix provides the detailed calculation of the optimal $c_{m}$.

Defining $Y=y y^{T}$, we can rewrite (32) as

$$
c_{m}=\sup _{Y \succeq 0} \frac{\operatorname{Tr}\left(\left(\boldsymbol{\Omega}_{e_{0}}^{T} \mathbf{Q}_{m} \boldsymbol{\Omega}_{e_{0}}-\rho_{m} \mathbf{Q}_{m}\right) Y\right)}{\operatorname{Tr}\left(\left(\boldsymbol{\Omega}_{e_{0}}^{T} \mathbf{Q}_{m} \boldsymbol{\Omega}_{e_{0}}-\boldsymbol{\Omega}_{e_{1}}^{T} \mathbf{Q}_{m} \boldsymbol{\Omega}_{e_{1}}\right) Y\right)} .
$$

We assume

$$
[Z]_{n, n}=1 / \operatorname{Tr}\left(\left(\boldsymbol{\Omega}_{e_{0}}^{T} \mathbf{Q}_{m} \boldsymbol{\Omega}_{e_{0}}-\boldsymbol{\Omega}_{e_{1}}^{T} \mathbf{Q}_{m} \boldsymbol{\Omega}_{e_{1}}\right) Y\right) .
$$

Then, (40) can be rewritten as [31]

$$
c_{m}^{\prime}=\sup _{Z \in \mathbb{R}^{n \times n},} \operatorname{Tr}\left(\left(\boldsymbol{\Omega}_{e_{0}}^{T} \mathbf{Q}_{m} \boldsymbol{\Omega}_{e_{0}}-\rho_{m} \mathbf{Q}_{m}\right) Z\right)
$$

s.t.

$$
\begin{aligned}
& \operatorname{Tr}\left(\left(\boldsymbol{\Omega}_{e_{0}}^{T} \mathbf{Q}_{m} \boldsymbol{\Omega}_{e_{0}}-\boldsymbol{\Omega}_{e_{1}}^{T} \mathbf{Q}_{m} \boldsymbol{\Omega}_{e_{1}}\right) Z\right)=1, \\
& Z \succeq 0,
\end{aligned}
$$

which is a typical semidefinite programming problem (SDP), and can be easily solved by available concave optimization [33]-[35]. Once (34) is solved, we can obtain the optimal $Z^{*}$ and $\left(c_{m}^{\prime}\right)^{*}$. Then, the solution for (40) can be obtained by

$$
\begin{aligned}
Y^{*} & =Z^{*} \cdot 1 / \operatorname{Tr}\left(\left(\boldsymbol{\Omega}_{e_{0}}^{T} P_{0} \boldsymbol{\Omega}_{e_{0}}-\boldsymbol{\Omega}_{e_{1}}^{T} P_{0} \boldsymbol{\Omega}_{e_{1}}\right) Y^{*}\right) \\
& =\frac{Z^{*}}{\left[Z_{n, n}^{*}\right]},
\end{aligned}
$$

and $c_{m}^{*}$ can be obtained accordingly.

\section{APPENDix B}

This appendix provides the proof that (37a) is a concave function with respect to $N_{m}$.

Given $p_{m}, \eta_{m}$ is a function with respect to $N_{m}$, where $N_{m} \geq 1$. From (36), we have

$$
\begin{aligned}
\eta_{m} & =\frac{\lambda}{N_{m}}\left(1-f_{Q}\left(\frac{N_{m} T_{t h} B_{0} C_{m}-\lambda+\log \left(N_{m} T_{t h} B_{0}\right) / 2}{(\log e) \sqrt{N_{m} T_{t h} B_{0}}}\right)\right) \\
& =f_{1}\left(N_{m}\right) \cdot f_{2}\left(N_{m}\right),
\end{aligned}
$$

where $f_{1}\left(N_{m}\right)=\frac{\lambda}{N_{m}}$, and

$$
f_{2}\left(N_{m}\right)=1-f_{Q}\left(\frac{N_{m} T_{t h} B_{0} C_{m}-\lambda+\log \left(N_{m} T_{t h} B_{0}\right) / 2}{(\log e) \sqrt{N_{m} T_{t h} B_{0}}}\right) .
$$

Property 4: Given $p_{m}, f_{2}\left(N_{m}\right)$ is a concave function with respect to $N_{m}$.

Proof: Since $h_{m}$ is constant during one packet transmission, we assume $\left|h_{m}\right|^{2}=1$ to simplify the proof. Assuming $G_{1}=$ $T_{t h} B_{0}, G_{2}=G_{1} \cdot \log \left(1+\frac{g_{m} p_{m}}{N_{0} B_{0}}\right)$, and $G_{3}=(\log e) \sqrt{G_{1}}$, then (44) can be rewritten as

$$
f_{2}\left(N_{m}\right)=1-f_{Q}\left(\frac{N_{m} G_{2}-\lambda_{m}+\log \left(N_{m} G_{1}\right) / 2}{G_{3} \sqrt{N_{m}}}\right) .
$$

Let

$$
f_{3}\left(N_{m}\right)=\frac{N_{m} G_{2}-\lambda_{m}+\log \left(N_{m} G_{1}\right) / 2}{G_{3} \sqrt{N_{m}}},
$$

then we take derivative with respect to $N_{m}$ in (47) and can obtain

$$
\begin{aligned}
& \frac{\partial f_{3}\left(N_{m}\right)}{\partial N_{m}}=\frac{G_{3}}{2 N_{m}^{\frac{1}{2}}} . \\
& \left(G_{1} \log \left(1+\frac{g_{m} p_{0}}{N_{0} B_{0}}\right) N_{m}+\left(1+\lambda_{m}\right)-\frac{\log \left(G_{1} N_{m}\right)}{2}\right) \\
& =f_{4}\left(N_{m}\right) \cdot f_{5}\left(N_{m}\right)
\end{aligned}
$$

where $f_{4}\left(N_{m}\right)=\frac{G_{3}}{2 N_{m}^{\frac{1}{2}}}$, and

$f_{5}\left(N_{m}\right)=G_{1} \log \left(1+\frac{g_{m} p_{0}}{N_{0} B_{0}}\right) N_{m}+\left(1+\lambda_{m}\right)-\frac{\log \left(G_{1} N_{m}\right)}{2}$.

In (48), it is obvious that $f_{4}\left(N_{m}\right)>0$ for any $N_{m}>0$. In (49), by taking derivative with respect to $N_{m}$, we can obtain

$$
\frac{\partial f_{5}\left(N_{m}\right)}{\partial N_{m}}=G_{1} \log \left(1+\frac{g_{m} p_{m}}{N_{0} B_{0}}\right)-\frac{1}{2 N_{m}} .
$$

Let $\frac{\partial f_{5}\left(N_{m}\right)}{\partial N_{m}}=0$, then we have

$$
N_{m, 0}=\frac{1}{2 G_{1} \log \left(1+\frac{g_{m} p_{m}}{N_{0} B_{0}}\right)} .
$$

Apparently, we have $N_{m, 0}<1$ in (51). In addition, $\frac{\partial f_{5}\left(N_{m}\right)}{\partial N_{m}}$ in (50) increases monotonically with $N_{m}$. Thus, $\frac{\partial f_{5}\left(N_{m}\right)}{\partial N_{m}}>0$ when $N_{m} \geq 1$, which means that $f_{5}\left(N_{m}\right)$ increases monotonically with $N_{m}$. This leads to $f_{5}\left(N_{m}\right)>0$ when $N_{m} \geq 1$. Thereby, we can obtain $\frac{\partial f_{3}\left(N_{m}\right)}{\partial N_{m}}>0$ when $N_{m} \geq 1$ in (48), which means that $f_{3}\left(N_{m}\right)$ increases monotonically with $N_{m}$. Furthermore, since $f_{Q}(\cdot)$ decreases monotonically, we can obtain the Q-function in (46) decreases monotonically with respective to $N_{m}$. In addition, $f_{Q}(\cdot)$ is a convex function. Thus, $f_{2}\left(N_{m}\right)$ is a concave function.

We have that $f\left(N_{m}\right)=f_{1}\left(N_{m}\right) \cdot f_{2}\left(N_{m}\right)$ is the perspective function of $f_{2}\left(N_{m}\right)$. Then, $f\left(N_{m}\right)$ is a concave function with respect to $N_{m}$ [33].

\section{REFERENCES}

[1] X. Li, Y. Yu, G. Sun, and K. Chen, "Connected vehicles' security from the perspective of the in-vehicle network," IEEE Network, vol. 32, no. 3, pp. 58-63, Jun. 2018.

[2] Y. Sadi and S. C. Ergen, "Optimal power control, rate adaptation, and scheduling for UWB-based intravehicular wireless sensor networks," IEEE Trans. Vehicular Technology, vol. 62, no. 1, pp. 219-234, Jan. 2013. 
[3] P. Wang, B. Di, H. Zhang, K. Bian, and L. Song, "Energy efficient V2Xenabled communications in cellular networks," IEEE Trans. Vehicular Technology, vol. 68, no. 1, pp. 554-564, Jan. 2019.

[4] S. Li, S. Xu, X. Huang, B. Cheng, and H. Peng, "Eco-departure of connected vehicles with V2X communication at signalized intersections," IEEE Trans. Vehicular Technology, vol. 64, no. 12, pp. 5439-5449, Dec. 2015.

[5] C. She, C. Yang, and T. Q. S. Quek, "Uplink transmission design with massive machine type devices in tactile internet," IEEE Globecom Workshops (GC Wkshps), Dec. 2016, pp. 1-6.

[6] C. She, C. Yang, and T. Quek, "Cross-layer optimization for ultrareliable and low-latency radio access networks," IEEE Trans. Wireless Commun., vol. 17, no. 1, pp. 127-141, Jan. 2018.

[7] C. Sun, C. She, C. Yang, T. Quek, Y. Li, and B. Vucetic "Optimizing resource allocation in the short blocklength regime for ultra-reliable and low-latency communications," IEEE Trans. Wireless Commun., vol. 18, no. 1, pp. 402-415, Jan. 2019.

[8] V. N. Swamy, et al., "Real-time cooperative communication for automation over wireless," IEEE Trans. Wireless Commun., vol. 16, no. 11, pp. 7168-7183, Nov. 2017.

[9] Y. Yu, H. Chen, Y. Li, Z. Ding, and B. Vucetic, "On the performance of non-orthogonal multiple access in short-packet communications," IEEE Commun. Lett., vol. 22, no. 3, pp. 590-593, Dec. 2017.

[10] H. V. Mendis and F. Li, "Achieving ultra reliable communication in 5G networks: a dependability perspective availability analysis in the space domain," IEEE Commun. Lett., vol. 21, no. 9, pp. 2057-2060, Sep. 2017.

[11] E. Dosti, U. Wijewardhana, H. Alves, and M. Latva-aho, "Ultra reliable communication via optimum power allocation for type-I ARQ in finite block-length," IEEE Inter. Conf. Commun. (ICC), May 2017, pp. 1-6.

[12] K. Trillingsgaard and P. Popovski, "Downlink transmission of short packets: framing and control information revisited," IEEE Trans. Commun., vol. 65, no. 5, pp. 2048-2061, May 2017.

[13] Y. Chen, L. Cheng, and L. Wang, "Prioritized resource reservation for reducing random access delay in 5G URLLC," IEEE Annual Inter. Symposium Personal, Indoor, and Mobile Radio Communications (PIMRC), Oct. 2017, pp. 1-5.

[14] R. Kotaba, C. Manchón, T. Balercia, and P. Popovski, "Uplink transmissions in URLLC systems with shared diversity resources," IEEE Wireless Commun. Letters, pp. 1-4, Jan. 2018.

[15] L. Schenato, B. Sinopoli, M. Franceschetti, K. Poola, and S. Sastry, "Foundations of control and estimation over lossy networks," IEEE Proc., vol. 95, no. 1, pp. 163-187, Jan. 2007.

[16] A. Cetinkaya, H. Ishii, and T. Hayakawa, "Networked control under random and malicious packet losses," IEEE Trans. Automatic Control, vol. 62, no. 5, pp. 2434-2449, Sep. 2017.

[17] Y. Feng, X. Chen, and G. Gu, "Output feedback stabilization for discretetime systems under limited communication," IEEE Trans. Automatic Control, vol. 62, no. 4, pp. 1927-1932, Apr. 2017.

[18] L. Su and G. Chesi, "On the robust stability of uncertain discrete-time networked control systems over fading channels," American Control Conference (ACC), Jul. 2015, pp. 6010-6015.

[19] L. Shi, Y. Yuan, and J. Chen, "Finite horizon LQR control with limited controller-system communication," IEEE Trans. Automatic Control, vol. 62, no. 7, pp. 1835-1841, Jul. 2013.

[20] P. Park, S. Ergen, C. Fischione, C. Lu, and K. Johansson, "Wireless network design for control systems: a survey," IEEE Commun. Surveys \& Tutorials, pp. 978-1013, Secondquarter 2017.

[21] Y. Polyanskiy, H. V. Poor, and S. Verdu, "Channel coding rate in the finite blocklength regime," IEEE Trans. Inf. Theory, vol. 56, no. 5, pp. 2307-2359, May 2010.

[22] G. Durisi, T. Koch, and P. Popovski, "Toward massive, ultrareliable, and low-latency wireless communication with short packets," IEEE Proc., vol.104, no. 9, pp. 1711-1726, Aug. 2016.

[23] G. Zhao, M. A. Imran, Z. Pang, Z. Chen, and Liying Li, "Toward realtime control in future wireless networks: communication-control codesign,” IEEE Commun. Magazine, vol.57, no. 2, pp. 138-144, Feb. 2019.

[24] P. Park, J. Araújo, and K. H. Johansson, "Wireless networked control system co-design," IEEE Inter. Conf. Networking, Sensing and Control (ICNSC), 2011, pp.486-491.

[25] B. Chang, G. Zhao, M. Imran, Zhi Chen, and L. Li "Dynamic wireless QoS analysis for real-time control in URLLC," IEEE Globecom Workshops (GC Wkshps), Dec. 2018, pp. 1-5.

[26] T. Zeng, O. Semiari, W. Saad, and M. Bennis, "Integrated communications and control co-design for wireless vehicular platoon systems," IEEE Inter. Conf. Commun. (ICC), May 2018, pp.1-6.
[27] A. Firooznia, J. Ploeg, N. Wouw, and H. Zwar, "Co-Design of controller and communication topology for vehicular platooning," IEEE Trans. Intelligent Transportation Systems, vol. 18, no. 10, pp. 2728-2739, Otc. 2017.

[28] 3GPP, Study on Scenarios and Requirements for Next Generation Access Technologies. Technical Specification Group Radio Access Network, Technical Report 38.913, Release 14, Oct. 2016.

[29] W. Yang, G. Durisi, T. Koch, and Y. Polyanskiy, "Quasi-static multipleantenna fading channels at finite blocklength," IEEE Trans. Inf Theory, vol. 60, no. 7, pp. 4232-4264, Jul. 2014

[30] J. Sarangapani and H. Xu, Optimal networked control systems with MATLAB (automation and control engineering), CRC Press, 2015.

[31] C. Chi, W. Li, and C Lin, Convex optimization for signal processing and communications: from fundamentals to applications, CRC Press, 2017.

[32] K. Gatsis, M. Pajic, A. Ribeiro, and G. J. Pappas, "Opportunistic control over shared wireless channels," IEEE Trans. Automatic Control, vol. 60, no. 12, pp. 3140-3155, Dec. 2015.

[33] S. Boyd and L. Vandenberghe, Convex optimization, Cambridge University Press, 2004.

[34] S. Ruscheweyh and T. Small, Comment. math. helv., 1973.

[35] E. Chong and S. Żak, An introduction to optimization (4th Edition), Wiley, 2001. 